\title{
Development of Wireless Networking and Synchronization in a Health Monitoring System
}

\author{
Tomoki Mino ${ }^{\mathrm{a},}$, Takeshi Wada ${ }^{\mathrm{a}}$, Kazuhiro Aoki ${ }^{\mathrm{a}}$, Takushi Obata ${ }^{\mathrm{b}}$, Kiyoshi Hayakawa ${ }^{\mathrm{a}}$ \\ a Osaka Prefecture University College of Technology, 26-11 Saiwai-cho, Neyagawa-shi 572-8572, Japan \\ bHokkai-Gakuen University, 4-1-40 Asahi-cho, Sapporo-shi 062-8605, Japan \\ *Corresponding Author: hayakawa@ osaka-pct.ac.jp
}

\begin{abstract}
In Japan, deterioration of the infrastructures in 1970's becomes a social problem. They become lifelong duration due to be fixed and repaired. Therefore, it is important to check and manage infrastructures on a daily basis. However, there are two problems of the checking. One is a shortage of specialists of the checking, the other is place regulation of infrastructure such as the bridges and highways. Therefore, we are developing a health monitoring system. It allows an amateur to check the infrastructures with Fourier spectrum. We employ ARM \& FPGA board to measure and analyze Fourier spectrum and also employ XBee ZB to construct wireless mesh network. In addition, we develop a low cost vibrometer using piezo-electric devices. We have developed a testbed health monitoring system which consists of one base module and one measurement module. Indeed, we have to put many base and measurement modules on huge infrastructures in order to check damage of them. In the circumstances, the system needs time synchronization between the modules. In this paper, we will propose time synchronization method called MHRBS- $\alpha$ and evaluate the method by 7 modules. In the result, we achieved the time difference within $5 \mathrm{~ms}$.
\end{abstract}

Keywords: Embedded systems, IoT, Piezoelectric element, Health monitoring system, Sensor network.

\section{Introduction}

Most building structures, such as office buildings, bridges, high ways, and so on, were built in 1970's and 1990's in japan. Deterioration of the building structures in 1970's becomes a social problem. Conventionally, the infrastructures were demolished and rebuilt. However, affected by the recent Japanese economic slump, the infrastructures were repaired and fix. Furthermore, we require the durability and earthquake-proof of the infrastructures for large-scale disasters and increase in car traffic. Consequently, the infrastructures become lifelong duration.

In order to put it into practice, it is important to check and manage the infrastructures on a daily basis. Ordinary, the infrastructures are checked by looking and hammering test of specialists. But, there are two problems of the checking. One is a shortage of the specialists, the other is place regulation of infrastructure such as the bridges and highways. We have to develop a damage identification monitoring system by which everyone can check the building structures without the specialists of checking and hammering test of the infrastructures.

Therefore, many monitoring systems of infrastructure have been developed ${ }^{(1)-(4)}$. we have also developed the health monitoring system for a testbed bridge ${ }^{(4)}$. It allows us to find the place and degree of the damage by processing signals of vibration in it. We have developed a low cost vibrometer using piezo-electric devices which are put on the testbed system. A previous work shows that it is possible for us to find the place of the damage on the infrastructures by the vibrometer using piezo-electric devices ${ }^{(5)}$.

The monitoring system, however, consisted of only one measurement module and one base module. In order to put it on huge infrastructures, we have to increase the measurement and base modules. Therefore, we assume that topology of the sensor network is tree topology, such as Fig.1. We employ Zigbee as a sensor network in Fig.1. C, R, and E indicate coordinator, Routor, End device respectively in Fig.1. In this topology, connection between sensor nodes is changed according as the place on which they put. 
Furthermore, the beginning time of the measurement isn't synchronized. In this paper, we will propose a novel synchronization method that allows the system begin the measurement at the same time.

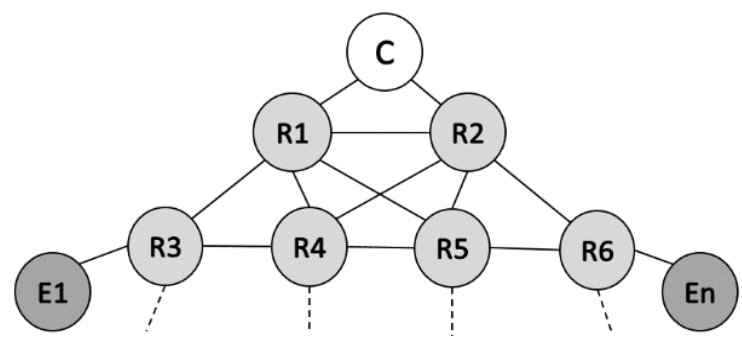

Fig. 1. Network topology on the proposed system

\section{The Proposed System}

Fig. 2 shows overall structure of the proposed system. It consists of measurement modules, a base module, and a cloud server. The measurement modules and the base module are set on the infrastructures such as a bridge described by Fig.2. They construct tree network automatically. The measurement modules measure vibration several times a day at starting signals from the base module. They also transform a vibration signal to a Fourier spectrum, and send the Fourier spectrum to the base modules. The base module sends Fourier spectrums received from many measurement modules to the cloud server by Ethernet.

It manages data of the infrastructures in all of our country, and finds damage of them. We are able to check Fourier spectrum and results of damage identification through Web application.

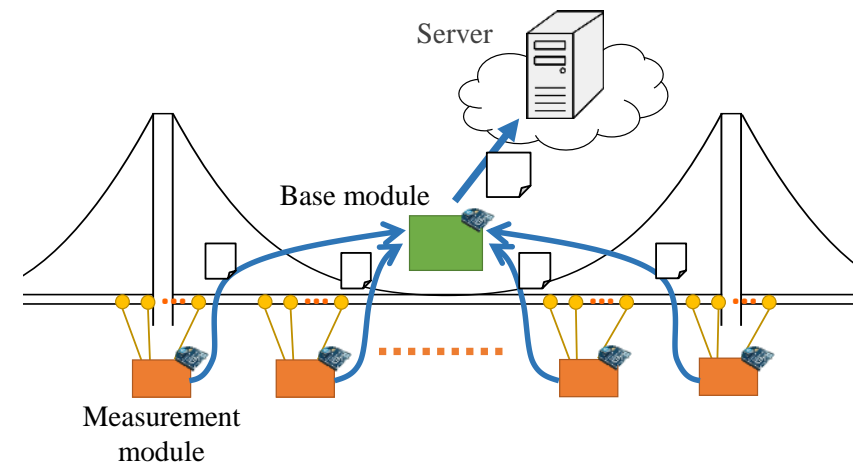

Fig. 2. Overall structure of the proposed system

\subsection{Measurement Modules}

Fig. 3 shows Organization of the measurement module. It consists of eight piezo-electric devices, an external A/D converter board, a ZYBO FPGA board, and a synchronization module.

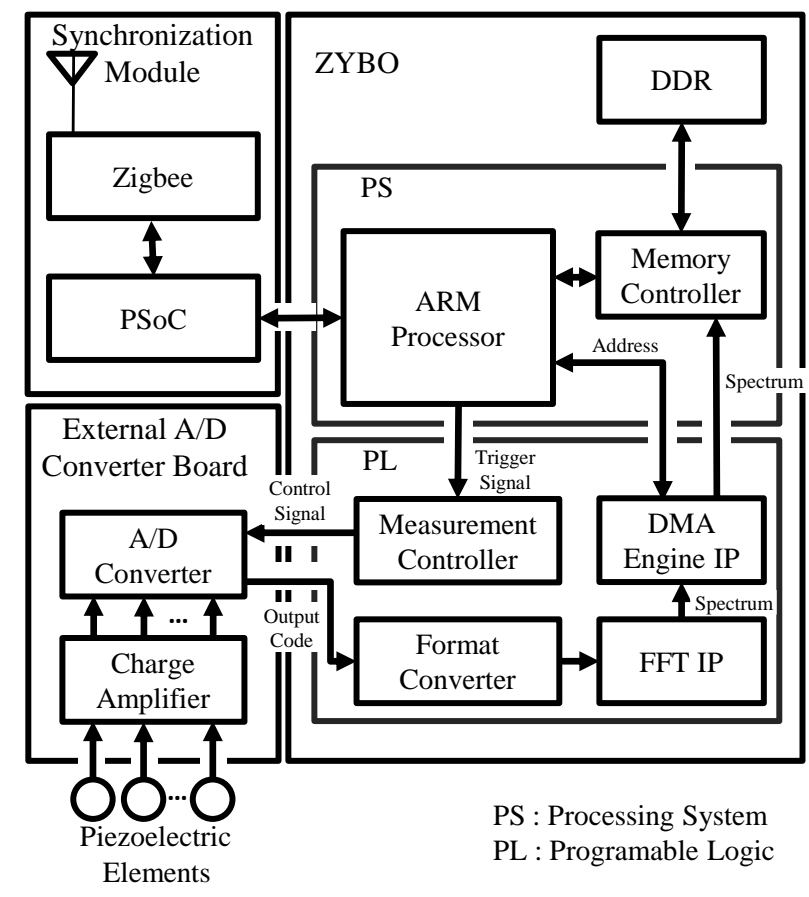

Fig. 3. Organization of the measurement modules

The external A/D converter board consists of a charge amplifier and A/D converter chip. It converts amount of electric charge generated by a piezo-electric device to voltages. And it also converts analog value of the voltages to digital value.

The measurement controller controls an MP3208 A/D converter chip which is able to convert 8 analog values simultaneously. When the ARM processors begin to measure, they send a trigger signal to the measurement controller. It receives the trigger signal and samples the vibration data of the infrastructures in the selected sampling clock with the MCP 3208. The MCP3208 sends the sampling data to format converter via SPI.

The format converter converts the sampling data into a format decided by the FFT IP. When it has finished converting all 8 sampling data, send them to the FFT IP.

We employ LogiCORE (FFT Logi CORE) produced by Xilinx inc as the FFT IP. It calculates discrete Fourier Transform with hardwired logic. FFT IP consists of switch, selector, data RAM, and butterfly computation logic. Butterfly logic employs Radix-2 algorithm (also known as the Cooley-Tukey algorithm). Radix 2 means that the number of samples must be an integral power of two. The decimation in time means that the algorithm performs a 
subdivision of the input sequence into its odd and even members.

The FFP IP writes the Fourier spectrum data to DDR memory via the DMA Engine IP. It interrupts ARM cores to inform the completion of the writing when it has finished writing the Fourier spectrum data.

\subsection{Base Module}

Fig. 4 shows organization of the base module. It consists of an mbed and a synchronous module. The mbed collects a lot of Fourier spectrum data from many measurement modules. In order to reduce wire cost of a network between base and measurement modules, we employ Xbee ZB wireless communication device in the synchronous module. The collected data was stored in a flash memory of the mbed temporarily. Once the base module has all the collected data, it sends the collected data to the cloud server.

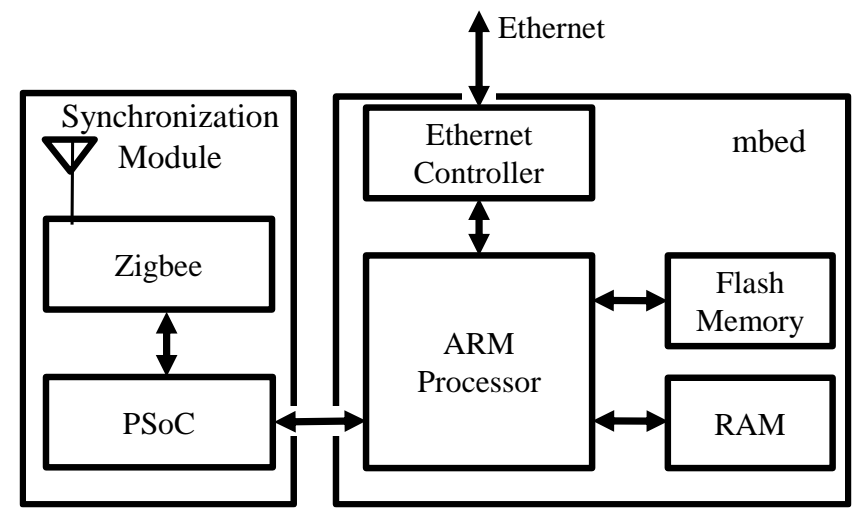

Fig. 4. Organization of base module.

\subsection{Synchronous module}

Fig.5 shows organization of the monitoring system including the synchronization module. The synchronization module enables time synchronization and automatically construction of sensor network.

We employ a PSoC (CY8C29466-24PXI) produced by Cypress corporation as micro controller, and an XBee ZB produced by Digi International corporation as wireless communication module.

The base module and the measurement modules communicate with each other via the synchronous module. The synchronous module sends the Fourier spectrum data via XBee wireless device.

The synchronization modules consist of parent modules and child modules. The parent modules control the child modules in order to enable time synchronization. The child modules synchronize with another child modules by out proposed synchronization method. In order to count the time, the child modules have crystal oscillator produced by Miyazaki Epson (TC0-707F).

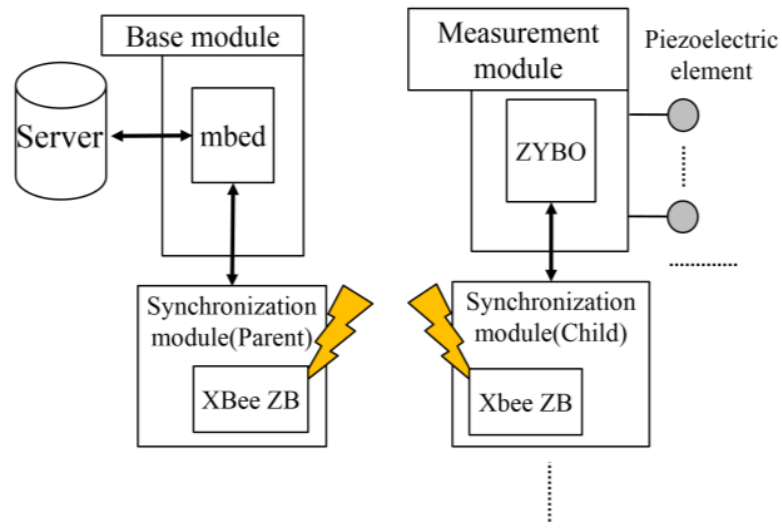

Fig. 5. Organization of system including the synchronization modules

\section{MHRBS method}

\subsection{RBS Method}

RBS (Reference Broadcast Synchronization) has been proposed as one of synchronization on sensor network ${ }^{(7)}$. RBS is used by time synchronization between proximity nodes in wireless network.

We describe RBS protocol in Fig.6. A wireless network consists of one parent node $(C)$ and 4 child nodes(R). The parent node connects to all child nodes, and child nodes connect with next to the child nodes (i.e. R3 connects with $\mathrm{R} 2$ and R4). The RBS protocol is below.

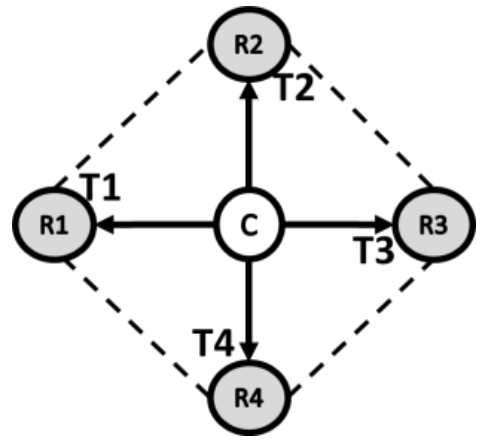

Fig. 6. Description of RBS (one parent and 4 child).

(1) A parent nodes(C) broadcasts a sync signal to all child node $(\operatorname{Rk}(\mathrm{k}=1,2, . ., \mathrm{N}) \mathrm{N}$ define the number of child nodes).

(2) All child nodes store time when a sync signal was received. 
(3) A child node exchanges the receive time with proximity nodes and revise the time according to Equation (1). That is, RBS adds $\theta$ to the present time.

$$
\theta=\frac{1}{N} \sum_{i=1}^{N}\left(T_{0}-T_{i}\right)
$$

$\mathrm{T}_{0}$ : Sync signal receive time

$\mathrm{T}_{\mathrm{i}}$ : Sync signal receive time from proximity nodes

$\mathrm{N}$ : Total of proximity nodes

$\theta$ : correction value

For example, R1 exchange the receive time with $\mathrm{R} 2$ and R4. R1 calculates $\theta=\left\{\left(\mathrm{T}_{0}-\mathrm{T}_{1}\right)+\left(\mathrm{T}_{0}-\mathrm{T}_{2}\right)\right\} / 2$ and add $\theta$ to the present time. Where, $\mathrm{T}_{1}$ indicates receive time of $\mathrm{R} 2$ and $\mathrm{T}_{2}$ indicates that of R4.

RBS is effective method for direct connection between proximity nodes. RBS for static multi hop network (i.e. indirect network) is proposed. That is, RBS is able to be employed a multi hop network which has already decided. But it is difficult that RBS is employed in dynamic multi hop network. Since Zigbee network is changed dynamically and automatically, we don’t employ RBS.

\subsection{MHRBS- $\alpha$ Method}

In this section, we describe MHRBS- $\alpha$. It is an improvement version of MHRBS (Multi-Hop RBS) which has been proposed by reference (4). MHRBS enable RBS in the dynamic multi hop network. In previous work, MHRBS has achieved synchronization which the time difference is within $5 \mathrm{~ms}$ in 5 synchronization modules. But it hasn't achieved synchronization which the time difference is within $5 \mathrm{~ms}$ over 7 synchronization modules. In this section, we propose MHRBS- $\alpha$ method. It synchronizes the beginning time of a measurement with 7 synchronization modules within $5 \mathrm{~ms}$ time difference.

\subsubsection{Protocol of MHRBS- $\alpha$}

In order to describe MHRBS- $\alpha$, we assumed that a multi hop network of the monitoring system is tree topology network like Fig.7. The nodes are classified as $\mathrm{C}$ (coordinator), R (router), and E (end device). We set Rank to the tree topology form 0 (root) to $\mathrm{k}$ (End device). The child nodes which have direct connection to the parent node must connect each other. That is, since R1 and R2 have direct connection to $\mathrm{C}, \mathrm{R} 1$ must connect to $\mathrm{R} 2$ directly. protocol is blow.
(1) C (the root node) sends a time reset signal to child nodes which is connected directly (nodes of Rank 1). We assumed that nodes of Rank 1 have received the time reset signal at the same time. They reset an internal clock to 0 and complete the synchronization between nodes of rank 1 .

(2) $\mathrm{C}$ sends 2 signals to a node of $\operatorname{Rank} \mathrm{k}(\mathrm{k}=1 . . \mathrm{j})$. The node of Rank k defines as Ro. One is search signal which

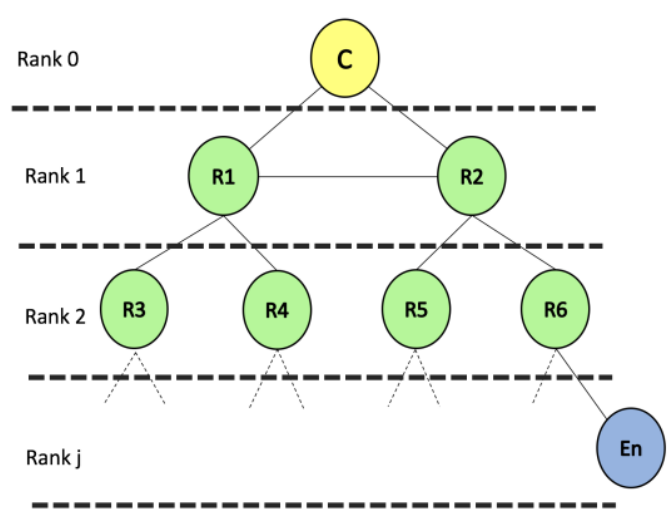

Fig.7 A multi hop network on the monitoring system

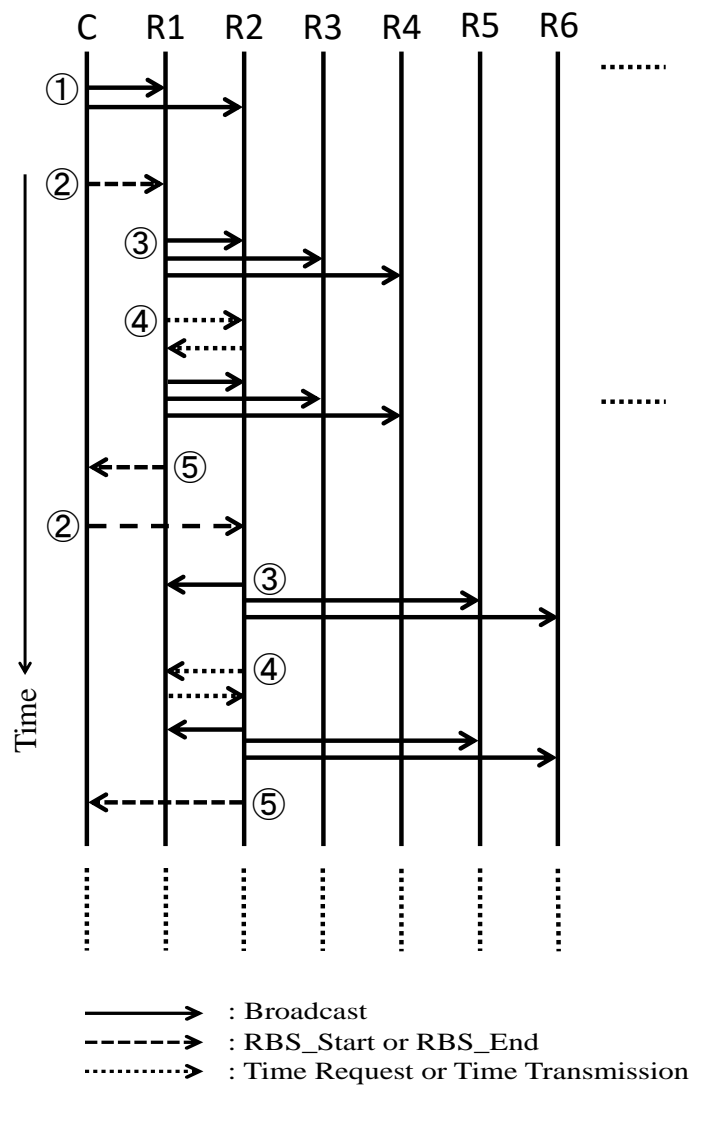

Fig.8 Communication sequences of MHRBS- $\alpha$ 
indicate that Ro finds child nodes of Rank $k+1$. The other is RBS_start signal which indicate that Ro performs RBS time synchronization among the child nodes and synchronized nodes. In case that Ro belongs to Rank 1, the synchronized node means nearby node of the same rank, and in case that Ro doesn't belong to Rank 1, the synchronized node means parent node which belongs to Rank k-1.

(3) Ro sends sync signal to the child nodes and the synchronized node which were found by protocol (2). The child nodes is defined as $\mathrm{Rp}$ ( $\mathrm{p}=$ the child nodes number: $\mathrm{p}=1 . . \mathrm{n})$. The synchronized node is defined as $\mathrm{Rq}(\mathrm{q}=$ synchronized node number: $\mathrm{q}=1 . . \mathrm{n}) . \quad \mathrm{Rp}$ and $\mathrm{Rq}$ store the received time of sync signal. The received time is defined as $T_{p}$ and $T_{q} . T_{q}$ is a base time.

(4) Ro receives the base time ( $\left.T_{q}\right)$ from Rq. Ro sends $T_{q}$ to $\mathrm{Rp}$ and $\mathrm{Rq}$ again. $\mathrm{Rp}$ and $\mathrm{Rq}$ store a received time of $T_{q}$. The received time is defined as $T_{p}^{\prime}$ and $T_{q}^{\prime}$. The recived time of $\mathrm{Rp}$ is defined as $T_{p}^{\prime}$. The recived time of $\mathrm{Rq}$ is defined as $T_{q}^{\prime}$. In our monitoring system, since $\mathrm{Rq}$ counts up timer slower than Rp due to interruption, we found a constant delay $(\alpha)$ in the $T_{q}^{\prime}$. We revise $T_{q}^{\prime}$ using Equation (2). Revised time is defined as $T_{q_{-} \alpha}^{\prime}$. Rq corrects timer value $\left(T_{q}^{+}\right)$using Equation (3), and Rp also corrects timer value $T_{p}^{+}$using Equation (4), $T_{m}^{\prime}$ defined as current time of child nodes number $m$.

$$
\begin{gathered}
T_{q_{-} \alpha}^{\prime}=T_{q}^{\prime}+\alpha \\
T_{q}^{+}=T_{q_{-} \alpha}^{\prime}-\left(T_{q}-T_{q}\right) \\
T_{p}^{+}=T_{p}^{\prime}-\left(\begin{array}{ll}
T_{p}-T_{q}
\end{array}\right)
\end{gathered}
$$

(5) Since Ro to $\mathrm{Rp}$ have finished synchronization, Ro sends RBS_end signal to C.

(6) C applies above protocol (2) - (5) to nodes of Rank k, $\mathrm{k}+1$, and $\mathrm{k}+2$. Ro belongs to Rank $\mathrm{k}+1, \mathrm{Rq}$ belongs to Rank k, and Rp belongs to Rank k+2.

For example, Fig.8 shows communication sequences of MHRBS- $\alpha$. For first sequence, Ro corresponds to R1, Rq corresponds to R2, and Rp corresponds to R3 and R4. For second sequence, Ro corresponds to R2, Rq corresponds to $\mathrm{R} 1$, and Rp corresponds to R5 and R6.

\section{Experiments}

\subsection{Experiment environment}

In order to evaluate effectiveness of MHRBS- $\alpha$, we measured the time difference between each $T_{m}^{+}$. Table 1 shows experiment environment on the monitoring system. In this experiment, we didn't use base and measurement modules due to evaluate only synchronization. We measured the constant delay $(\alpha)$ in advance. In this result, $\alpha$ defined $1.8 \mathrm{~ms}$. A communication interruption takes place the constant delay in the synchronized node. We estimated time of the interruption at $1.6 \mathrm{~ms}$.

Table 1 Experiment environment

\begin{tabular}{|c|c|}
\hline Num. of nodes & $7(\mathrm{C}=1, \mathrm{R}=6)$ \\
\hline Method & MHRBS and MHRBS- $\alpha$ \\
\hline Conditions & Sync modules \\
\hline Target value & Within 5ms \\
\hline$\alpha$ & $1.8 \mathrm{~ms}$ \\
\hline
\end{tabular}

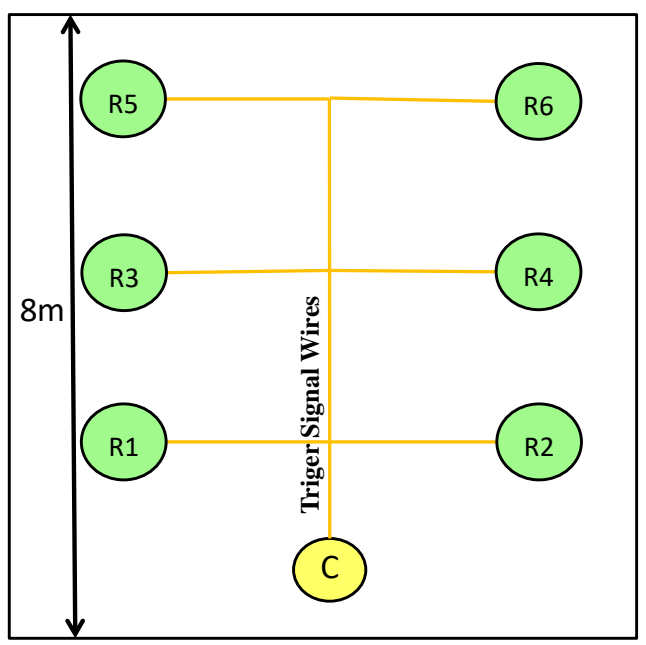

Fig.9 Arrangement of the nodes

Fig.9 shows the position of the nodes that have been arranged. In order to read internal clock of each $\mathrm{R}$ nodes, we must have access to $\mathrm{R}$ nodes. Therefore, we provided a trigger signal wires. It links $\mathrm{C}$ and $R m(\mathrm{~m}: 1-6)$. $\mathrm{C}$ triggers a collection of $T_{m}^{+}$by the wire after synchronization is over. Then each $R m$ nodes send $T_{m}^{+}$to $\mathrm{C}$ via Xbee ZB (Zigbee wireless communication).

\subsection{Results of the experiment}

We have already mentioned that Zigbee network is changed dynamically and automatically. In the experiment, Zigbee nodes construct network like Fig.10 automatically. C connected R1, R2, and R3 directly. Furthermore, R1, R2, and $\mathrm{R} 3$ connected each other ( complete link).

Table 2 shows results of time difference in MHRBS and MHRBS- $\alpha$. We measured $T_{m}^{+}$at 15 times and calculated the 
time difference between them. In 15 times data, MHRBS (old version) achieved that maximum difference was 7.9[ms], minimum difference was $5.9[\mathrm{~ms}]$ On the other hand, MHRBS- $\alpha$ achieved that maximum difference was 3.2[ms], minimum difference was $0.6[\mathrm{~ms}]$. MHRBS- $\alpha$ was less maximum time difference than MHRBS by $45.8[\%]$.

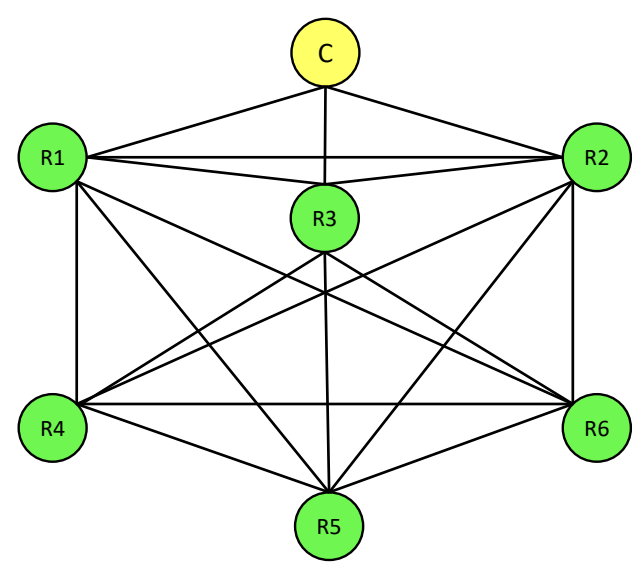

Fig.10 Result of network organization

Table 2 Results of the time difference

\begin{tabular}{|c|c|c|c|}
\hline & $\begin{array}{c}\text { Max } \\
{[\mathrm{ms}]}\end{array}$ & $\begin{array}{c}\text { Min } \\
{[\mathrm{ms}]}\end{array}$ & $\begin{array}{c}\text { Average } \\
{[\mathrm{ms}]}\end{array}$ \\
\hline MHRBS & 7.9 & 5.9 & 7.1 \\
\hline MHRBS- $\alpha$ & 3.2 & 0.6 & 1.8 \\
\hline
\end{tabular}

We refer to correction value $\alpha$. A cause of $\alpha$ assumes that the base time may not count precisely. the synchronization module sends an interrupt signal to a processor which is embedded in mbed or ZYBO at stated periods using the crystal oscillator. In the interrupt, the processor counts the clock at stated periods. But the node which sends the base time fails to receive the interrupt signal in MHRBS- $\alpha$ protocol (4). That is, the node can't receive the interrupt signal during the node sends the base time and fails to count the clock. The time difference increases when the node takes on sending the base time many times. In this result, MHRBS reaches the maximum time difference of $7.9 \mathrm{~ms}$.

When the role of transmission of the base time was assigned to different nodes, the time difference reduced. But MHRBS- $\alpha$ is superior to this above method in the reduction of the time difference.

\section{Conclusion}

In this paper, we proposed time synchronization method called MHRBS- $\alpha$. We introduced our monitoring system and organization of time synchronization hardware. We described RBS by which MHRBS is inspired and protocol of MHRBS- $\alpha$. In experiment results, MHRBS- $\alpha$ is able to reduce time difference by $45.8 \%$ and the time difference was within $5 \mathrm{~ms}$.

There are 2 future works. One is an increase in the number of synchronization modules. We made the experiment whose area is under less $10 \mathrm{~m}^{2}$. We will expand area over $100 \mathrm{~m}^{2}$ and increase the number of synchronization modules. The other is demonstration experiment of our provided monitoring system which synchronization module is included. We will find out whether or not the synchronization modules come into collision with other modules.

\section{References}

(1) Yuji Ishikawa, Kenichi Takemoto, and Sanae Miyazaki : "Development of Real Time Remote Monitoring System of Bridges", NTT Technical journal, Vol.18, No.9, pp.21-36,2006.

(2) Yicheng Tsai, Yoshihiro Nitta, Masami Ishida, Toshio Onai, Yumi Hoshino and Akira Nishitani : "Structural Health Monitoring Utilizing dsPIC : Part 1 Development of monitoring system utilizing dsPIC", Summaries of technical papers of Annual Meeting Architectural Institute of Japan. B-2, Structures II, Structural dynamics nuclear power plants, 2009, pp. 659-660, 2009

(3) Yoshihiro Nitta, Masami Ishida, Yicheng Tsai, Toshio Onai, Akira Nishitani and Yumi Hoshino : "Structural Health Monitoring Utilizing dsPIC : Part 2 Pre-SHM Utilizing SFFT", Summaries of technical papers of Annual Meeting Architectural Institute of Japan. B-2, Structures II, Structural dynamics nuclear power plants, 2009, pp. 661-662, 2009

(4) Kiyoshi Hayakawa, Masashi Fujiwara, Takeshi Wada, and Takashi Obata : "Development of a damage identification monitoring system for building structures", the 4th IIAE International Conference on Industrial Application Engineering, pp.340-347, 2016

(5) Takashi Obata : "Experimental study of damage identification health monitoring system by using piezoelectric element", Journal of structural engineering", Vol. 60A, pp. 165-174, 2014

(6) J. Elson, L. Girod and D. Estrin: "Fine-Grained Network Time Synchronization using Reference Broadcasts", Proceedings of the 5th Symposium on 
Operating Systems Design and Implementation.

(7) Hiroshi Enokura, Kiyoshi Hayakawa, Takeshi Wada, Takushi Obata, "Development of the radio networking and measurement synchronization in a damage identification system", 2016 KOSEN convention record IEE Japan, pp.13-14, 2015 